\section{Presença de Centros de Especialidades Odontológicas e sua relação com a realização de exodontias na rede de atenção de saúde bucal no Brasil}

\section{Presence of Specialized Dentistry Centers and the relationship with dental extractions in the oral healthcare network in Brazil}

\author{
Presencia de Centros de Especialidades \\ Odontológicas y su relación con la realización \\ de exodoncias en la red de atención de \\ salud bucal en Brasil
}

\author{
Caroline Stein 1 \\ Karoline Weber dos Santos 1 \\ Aline Macarevich Condessa 1 \\ Roger Keller Celeste 1 \\ Juliana Balbinot Hilgert 1 \\ Fernando Neves Hugo 1
}

doi: 10.1590/0102-311X00054819

\section{Resumo}

Objetivou-se avaliar a associação entre presença de Centros de Especialidades Odontológicas (CEO) e a proporção de exodontias em relação ao total de procedimentos odontológicos em serviços públicos do Brasil. Trata-se de um estudo ecológico, em que foram avaliados dados sociodemográficos, dos serviços de saúde bucal e produção ambulatorial de 5.333 municípios nos biênios de 2000/2001 e 2015/2016. A principal variável de exposição foi a presença de CEO, e o desfecho a média nacional da proporção de exodontias em relação ao número de procedimentos odontológicos preventivos e curativos. Foram realizadas análises de interação e regressão múltipla usando modelo binomial com função de ligação logito. A média nacional da proporção de exodontias em relação aos procedimentos odontológicos preventivos e curativos foi $27,07 \%$ e 15,11\% em 2000/2001 e 2015/2016, respectivamente. Na análise de interação entre a presença de CEO e a cobertura superior a $80 \%$ de equipes de saúde bucal (ESB), houve menores proporções de exodontias em relação aos procedimentos odontológicos preventivos e curativos $(O R=0,71$; IC95\%: 0,71-0,72). Na análise de regressão múltipla, municípios com Índice de Desenvolvimento Humano entre 0,6-0,7 (OR = 0,77; IC95\%: 0,77-0,77), Produto Interno Bruto per capita maior que 20 mil Reais $(O R=0,45$; IC95\%: 0,45-045) e maior população residente em zona urbana (OR =0,72; IC95\%: 0,72-0,72) apresentaram menor proporção de exodontias em relação aos procedimentos odontológicos preventivos e curativos em 2015/2016. Conclui-se que ocorreram menores proporções de exodontias em relação aos procedimentos odontológicos preventivos e curativos em municípios com ao menos um CEO e com mais de 80\% de cobertura de ESB, o que aponta que municípios com Rede de Atenção à Saúde Bucal consolidada têm melhor desempenho na oferta de cuidados odontológicos.

Serviços de Saúde Bucal; Especialidades Odontológicas; Avaliação de Serviços de Saúde; Atenção Secundária à Saúde; Atenção Primária à Saúde

\section{Correspondência}

C. Stein

Universidade Federal do Rio Grande do Sul.

Rua Ramiro Barcelos 2492, Porto Alegre, RS 90035-004, Brasil. csteinodonto@hotmail.com

1 Universidade Federal do Rio Grande do Sul, Porto Alegre, Brasil. 


\section{Introdução}

A Política Nacional de Saúde Bucal (PNSB) tem como objetivo a reorganização dos serviços e saúde bucal nos níveis de atenção primária, especializada e hospitalar ${ }^{1}$. Para avaliar o desempenho do Sistema Único de Saúde (SUS) quanto ao cumprimento de seus princípios e diretrizes, instituiu-se o Índice de Desempenho do Sistema Único de Saúde (IDSUS), que tem entre seus indicadores de efetividade da atenção primária a proporção de exodontias em relação aos procedimentos odontológicos preventivos e curativos. O índice tem como objetivo estimular a redução do número de exodontias e aumentar o número de procedimentos conservadores e preventivos. O parâmetro de $8 \%$ do indicador de exodontias em relação aos procedimentos é utilizado pelo Ministério da Saúde para que os municípios se orientem a fim de melhorar a qualidade do cuidado em saúde bucal 2.

A ampliação e a qualificação da oferta de serviços especializados, previstas na PNSB, ocorreram em 2004 com o início da implantação dos Centros de Especialidades Odontológicas (CEO) e o estímulo à expansão à saúde bucal na Estratégia Saúde da Família (ESF), com intuito de constituir uma Rede de Atenção à Saúde Bucal (RASB) 1,3. Em 2004, o Brasil contava com 100 CEOs e, em 2017, este número aumentou para 1.033 serviços $1,4,5$. O CEO objetiva a integralidade da assistência à saúde bucal, sendo formado minimamente por cinco especialidades: periodontia, endodontia, cirurgia oral menor, diagnóstico bucal com ênfase no câncer bucal e atendimento a pacientes com necessidades especiais 6 .

Considerando o forte investimento do Ministério da Saúde na implantação de centenas de CEOs e o acompanhamento de indicadores de efetividade da atenção primária, faz-se necessária a avaliação desses centros quanto à sua contribuição para efetivação da integralidade na RASB, por meio da oferta de cuidados de maior densidade tecnológica. Da mesma forma, os municípios brasileiros que incorporaram equipes de saúde bucal (ESB) na saúde da família aumentaram as taxas de procedimentos coletivos, preventivos e restauradores, bem como reduziram a realização de exodontias 7,8. No entanto, não se encontraram evidências de que os CEOs implementados desde o começo da PNSB foram capazes de contribuir para o desempenho da RASB na perspectiva da integralidade do cuidado.

A hipótese do estudo é de que a ampliação dos serviços da atenção especializada por intermédio dos CEOs contribuiu para a melhora do cuidado à saúde bucal, com a diminuição de exodontias, o que ao mesmo tempo pode contribuir com a diminuição da necessidade de tratamentos reabilitadores protéticos no futuro. Assim, o objetivo deste estudo foi avaliar a associação entre presença de centros de especialidades odontológicos e a proporção de exodontias em relação ao total de procedimentos preventivos e curativos em serviços públicos de saúde dos municípios brasileiros, nos anos de 2000/2001 e 2015/2016.

\section{Métodos}

\section{Delineamento}

Trata-se de um estudo ecológico que incluiu os 5.552 municípios do Brasil com extração de dados realizada entre maio e setembro de 2017.

\section{Variáveis, fonte dos dados e mensuração}

O estudo teve como desfecho a média nacional da proporção de exodontias em relação ao número de procedimentos odontológicos preventivos e curativos realizados na atenção primária à saúde. Nesta proporção, o numerador foi composto pelas exodontias de decíduos e permanentes e o denominador pelos procedimentos preventivos (aplicação de flúor, selante e cariostático) e curativos (restaurações, tratamentos endodônticos, tratamentos periodontais e exodontias) (ver Material Suplementar: http://cadernos.ensp.fiocruz.br/site/public_site/arquivo/suppl-e00054819_8410.pdf). Para se obter a média nacional, foram calculadas as médias por município dos procedimentos realizados nos anos 2000 e 2001, considerado como um período de referência em relação à média de procedimentos em 2015 e 2016. Os procedimentos foram selecionados com base no indicador 21 do IDSUS: "Proporção de exodontia entre procedimentos odontológicos selecionados" (http://idsus.saude.gov.br/detalha 
das.html, acessado em 11/Jun/2017). Em função da alteração na tabela de procedimentos, foi necessário realizar uma adaptação do indicador. Antes de 2007, as restaurações eram contabilizadas por material e não por tipo de dente (decíduo ou permanente); optou-se pela inclusão dos procedimentos em decíduos para compor o indicador em ambos os períodos de análise (2000/2001 e 2015/2016).

A média de procedimentos realizados nos anos 2000 e 2001 foi utilizada como base para controle do desfecho (proporção de exodontias por procedimentos em 2015/2016). Para a coleta dos dados de 2000 e 2001, a página da Internet do Departamento de Informática do SUS (DATASUS; http:// datasus.saude.gov.br/) foi acessada, selecionando procedimentos por local de atendimento até 2007, todos os municípios, opção: procedimentos após 1999. Optou-se pela manutenção de todos os tipos de restauração, pois eram os materiais disponíveis na época.

A principal variável de exposição, presença de CEO, foi coletada no Cadastro Nacional de Estabelecimentos de Saúde (CNES) pela identificação do número de CEOs ativos nos municípios em dezembro de 2016, uma vez que o quantitativo de estabelecimentos é disponível apenas em relação aos centros ativos no ano corrente, tendo sido coletados dados relacionados ao município e tipo de CEO no município (I, II ou III).

Para compor o modelo de estudo, foram extraídos dados para as demais variáveis com anos próximos ao de interesse, 2015/2016, de acordo com a disponibilidade dos dados nos sistemas de informações. As variáveis e a fonte de dados estão apresentadas no Quadro 1. Para cada acesso de dados realizado, o banco de dados encontrado na página da Internet acessada foi baixado em formato Excel (Microsoft Corp.; https://products.office.com/) e se extraíram as variáveis de interesse que foram sendo combinadas (merged) pelo número de identificação do município pelo Instituto Brasileiro de Geografia e Estatística (IBGE) em um novo banco de dados que resultou no banco de dados completo para as análises do estudo.

\section{Análise estatística}

Foi realizada a descrição das variáveis por meio de média e desvio padrão, para as variáveis contínuas, e frequência absoluta e relativa para as variáveis categóricas. As análises bivariadas do desfecho com as demais covariáveis foram realizadas com teste de Kruskal-Wallis.

Estimativas de efeito foram calculadas por regressão múltipla com uso do modelo binomial com função de ligação logito, tendo o número de exodontias como variável dependente e o número total de procedimentos como denominador. Tal análise pondera os intervalos de confiança, dando maior peso para municípios que possuem maior número de procedimentos. Além disso, realizou-se análise de interação entre a presença de CEO e cobertura da ESB nos procedimentos de exodontias. Os dados foram analisados com o software estatístico Stata 13.1 (https://www.stata.com).

\section{Resultados}

Dos 5.552 municípios brasileiros, 5.333 (96\%) possuíam dados de procedimentos odontológicos a serem analisados e compuseram a amostra. Nos anos 2000/2001, 6,54\% (363) dos municípios não informaram nenhum procedimento de exodontia, e em relação aos demais municípios, a proporção de exodontias em relação aos procedimentos odontológicos preventivos e curativos foi de 27,07\%. Já em 2015/2016, 12,39\% (688) municípios não informaram exodontias, e os demais municípios apresentaram uma proporção de exodontias em relação aos procedimentos odontológicos preventivos e curativos de 15,11\%, não se ponderando o porte municipal em relação aos procedimentos realizados. Foram identificados 1.090 CEOs, distribuídos em 16,12\% (895) municípios brasileiros e a taxa de estabelecimentos em relação à população foi de 5,1 unidades/milhão de habitantes. Em relação ao cumprimento do indicador de efetividade da atenção primária por meio da proporção de exodontias em relação aos procedimentos odontológicos preventivos e curativos, apenas 16,79\% (932) dos municípios alcançaram o parâmetro estabelecido de até 8\% em 2000/2001, enquanto em 2015/2016 passou para 30,21\% (1.677).

Verificou-se que a média da proporção de exodontias em relação ao número de procedimentos odontológicos preventivos e curativos apresentou associação com todas as variáveis contextuais 
Quadro 1

Composição e fonte de dados das variáveis do estudo.

\begin{tabular}{|c|c|c|}
\hline VARIÁVEL & FONTE DOS DADOS & PÁGINA DA INTERNET ACESSADA \\
\hline \multicolumn{3}{|l|}{ Sociodemográficas e econômicas } \\
\hline Percentual da população entre 0 e 19 anos & $\begin{array}{l}\text { Atlas de Desenvolvimento Humano - } \\
\text { informações do ano de } 2010\end{array}$ & http://www.atlasbrasil.org.br/2013/pt/consulta/ \\
\hline Percentual da população feminina & $\begin{array}{l}\text { Atlas de Desenvolvimento Humano - } \\
\text { informações do ano de } 2010\end{array}$ & http://www.atlasbrasil.org.br/2013/pt/consulta/ \\
\hline Percentual de população urbana vs. rural & $\begin{array}{c}\text { DATASUS - Informações de Saúde (TABNET) - } \\
\text { Censo } 2010\end{array}$ & $\begin{array}{l}\text { http://www2.datasus.gov.br/DATASUS/index. } \\
\text { php?area=0206 }\end{array}$ \\
\hline Porte populacional dos municípios & $\begin{array}{c}\text { DATASUS - Informações de Saúde (TABNET) - } \\
\text { população estimada em } 2016\end{array}$ & $\begin{array}{c}\text { http://tabnet.datasus.gov.br/cgi/deftohtm. } \\
\text { exe?ibge/cnv/poptbr.def }\end{array}$ \\
\hline $\mathrm{IDH}$ & $\begin{array}{l}\text { Atlas de Desenvolvimento Humano - } \\
\text { informações do ano de } 2010\end{array}$ & http://www.atlasbrasil.org.br/2013/pt/consulta/ \\
\hline PIB municipal per capita & $\begin{array}{c}\text { DATASUS - Informações de Saúde (TABNET) - } \\
\text { média entre os anos } 2011 \text { a } 2013\end{array}$ & $\begin{array}{l}\text { http://www2.datasus.gov.br/DATASUS/index. } \\
\text { php?area=0206 }\end{array}$ \\
\hline \multicolumn{3}{|l|}{ Serviços de saúde } \\
\hline $\begin{array}{l}\text { Presença e tipo de CEO no município } \\
\text { (nenhum, I, II, III) }\end{array}$ & $\begin{array}{c}\text { CNES (DATASUS) - serviços ativos em } \\
\text { dezembro de } 2016\end{array}$ & $\begin{array}{l}\text { http://cnes.datasus.gov.br/pages/ } \\
\text { estabelecimentos/consulta.jsp }\end{array}$ \\
\hline Cobertura de equipe de saúde da família & $\begin{array}{c}\text { SIAB (DATASUS) - média entre os anos } 2015 \\
\text { e } 2016\end{array}$ & $\begin{array}{l}\text { http://tabnet.datasus.gov.br/cgi/deftohtm. } \\
\text { exe?cnes/cnv/equipebr.def }\end{array}$ \\
\hline Cobertura de equipe de saúde bucal & $\begin{array}{c}\text { SIAB (DATASUS) - média entre os anos } 2015 \\
\text { e } 2016\end{array}$ & $\begin{array}{c}\text { http://tabnet.datasus.gov.br/cgi/deftohtm. } \\
\text { exe?cnes/cnv/equipebr.def }\end{array}$ \\
\hline \multicolumn{3}{|l|}{ Produção ambulatorial } \\
\hline $\begin{array}{l}\text { Média nacional da proporção de } \\
\text { exodontias em relação ao número de } \\
\text { procedimentos odontológicos preventivos } \\
\text { e curativos (indicador de efetividade da } \\
\text { atenção primária à saúde, de acordo com o } \\
\text { Ministério da Saúde) }\end{array}$ & $\begin{array}{l}\text { SIA-SUS (DATASUS) - média municipal } \\
\text { 2000/2001 e } 2015 / 2016\end{array}$ & $\begin{array}{c}\text { http://tabnet.datasus.gov.br/cgi/deftohtm. } \\
\text { exe?sia/cnv/pabr.def }\end{array}$ \\
\hline
\end{tabular}

CEO: Centro de Especialidades Odontológicas; CNES: Cadastro Nacional de Estabelecimento de Saúde; DATASUS: Departamento de Informática do SUS; SIAB: Sistema de Informações em Atenção Básica; SIA-SUS: Sistema de Informações Ambulatoriais do SUS.

analisadas, bem como com a oferta de serviços odontológicos (Tabela 1). Na análise de regressão múltipla, para os fatores contextuais e oferta de serviços, observou-se que municípios com Índice de Desenvolvimento Humano (IDH) entre 0,6-0,7 (OR = 0,77; IC95\%: 0,77-0,77), Produto Interno Bruto (PIB) maior que 20 mil Reais (OR = 0,45; IC95\%: 0,45-045) e maior população residente em zona urbana (OR = 0,72; IC95\%: 0,72-0,72) apresentam menor proporção de exodontias em relação aos procedimentos odontológicos preventivos e curativos (Tabela 2). Além disso, quanto maior a proporção de população de jovens entre 0 e 19 anos, menor foi a realização desse procedimento.

$\mathrm{Na}$ análise de interação entre a presença de ao menos um CEO no município e a cobertura de ESB, constatou-se que houve menores proporções de exodontias em municípios com ao menos um CEO é potencializada quando a cobertura de ESB é superior a 80\% (OR =0,71; IC95\%: 0,71-0,72) (Tabela 3). 
Tabela 1

Análise da associação das variáveis socioeconômicas e oferta de serviços com a proporção de exodontias em relação aos procedimentos odontológicos preventivos e curativos nos municípios brasileiros em 2015/2016.

\begin{tabular}{|c|c|c|c|c|c|}
\hline \multirow[t]{2}{*}{ Variável } & \multicolumn{2}{|c|}{ Total } & \multirow{2}{*}{$\begin{array}{c}\text { Média da proporção } \\
\text { de exodontias }\end{array}$} & \multirow[t]{2}{*}{ DP } & \multirow[t]{2}{*}{ Valor de $p$} \\
\hline & $\mathbf{n}$ & $\%$ & & & \\
\hline População entre 0 e 19 anos (\%) & & & & & $<0,00$ \\
\hline$<30$ & 909 & 16,37 & 10,00 & 8,20 & \\
\hline $30-39$ & 3.410 & 61,41 & 14,10 & 12,70 & \\
\hline$>40$ & 1.233 & 22,20 & 21,70 & 16,80 & \\
\hline População feminina (\%) & & & & & $<0,00$ \\
\hline$<50$ & 1.778 & 32,02 & 16,60 & 15,00 & \\
\hline$>50$ & 3.774 & 67,97 & 14,40 & 13,00 & \\
\hline Porte populacional (habitantes) & & & & & $<0,00$ \\
\hline$<10.000$ & 2.430 & 43,77 & 13,63 & 12,17 & \\
\hline $10.000-50.000$ & 2.463 & 44,36 & 17,09 & 15,13 & \\
\hline$>50.000$ & 659 & 11,87 & 13,17 & 12,41 & \\
\hline População residente em zona urbana (\%) & & & & & $<0,00$ \\
\hline$<50$ & 1.608 & 28,96 & 17,79 & 16,01 & \\
\hline $50-75$ & 1.921 & 34,60 & 16,02 & 14,10 & \\
\hline$>75$ & 2.023 & 36,44 & 12,11 & 10,37 & \\
\hline IDH & & & & & $<0,00$ \\
\hline$<0.60$ & 1.391 & 25,05 & 21,94 & 16,96 & \\
\hline $0.60-0.70$ & 2.259 & 40,69 & 14,85 & 12,75 & \\
\hline$>0.70$ & 1.902 & 34,26 & 10,42 & 9,42 & \\
\hline PIB per capita (Reais) & & & & & $<0,00$ \\
\hline$>10.000$ & 2.383 & 42,92 & 19,06 & 16,0 & \\
\hline $10.000-20.000$ & 1.892 & 34,08 & 13,09 & 11,73 & \\
\hline$>20.000$ & 1.277 & 23,00 & 10,72 & 8,96 & \\
\hline Tipo de CEO no município & & & & & $<0,00$ \\
\hline Nenhum & 4.657 & 83,88 & 15,73 & 14,25 & \\
\hline 1 & 409 & 7,37 & 12,81 & 9,86 & \\
\hline II & 390 & 7,02 & 11,26 & 10,17 & \\
\hline III & 96 & 1,73 & 10,48 & 7,17 & \\
\hline Cobertura de EqSF e ESB (\%) & & & & & $<0,00$ \\
\hline$<80$ & 3.989 & 71,85 & 15,65 & 14,27 & \\
\hline$>80$ & 1.563 & 28,15 & 13,72 & 12,02 & \\
\hline Total & 5.552 & 100,00 & 15,11 & 13,70 & \\
\hline
\end{tabular}

CEO: Centro de Especialidades Odontológicas; DP: desvio padrão; ESB: equipe de saúde bucal; EqSF: equipe de saúde da família; IDH: Índice de Desenvolvimento Humano; PIB: Produto Interno Bruto.

\section{Discussão}

O presente estudo constatou que a presença de CEO no município esteve associada à menor média da proporção de exodontias em relação aos procedimentos preventivos e curativos, com melhores resultados onde a cobertura de ESB era maior que $80 \%$. Tais achados apontam para uma relação positiva entre a constituição da RASB e a efetivação de cuidados integrais à saúde bucal, sendo este o primeiro estudo a relacionar os serviços especializados com um menor número de procedimentos mutiladores no Brasil, de extrema importância para avaliação do desempenho da rede na perspectiva dos resultados. Dessa forma, sugere-se que a organização RASB esteja associada com menores proporções de realização de exodontias em relação aos demais procedimentos odontológicos. 
Tabela 2

Análises bruta e ajustada da associação das variáveis em estudo com a proporção de exodontias realizadas em relação aos procedimentos odontológicos preventivos e curativos nos municípios brasileiros em 2015/2016.

\begin{tabular}{|c|c|c|c|c|}
\hline \multirow[t]{2}{*}{ Variável } & \multicolumn{2}{|c|}{ Análise bruta } & \multicolumn{2}{|c|}{ Análise ajustada } \\
\hline & OR & IC95\% & OR & IC95\% \\
\hline \multicolumn{5}{|c|}{ População entre 0 e 19 anos (\%) } \\
\hline$<30$ & 1,00 & & 1,00 & \\
\hline $30-39$ & 1,77 & $1,77-1,77$ & 1,24 & $1,24-1,24$ \\
\hline$>40$ & 2,66 & $2,66-2,67$ & 1,16 & $1,16-1,16$ \\
\hline \multicolumn{5}{|c|}{ Usuárias mulheres (\%) } \\
\hline$<50$ & 1,00 & & 1,00 & \\
\hline$>50$ & 0,63 & $0,63-0,63$ & 0,83 & $0,83-0,83$ \\
\hline \multicolumn{5}{|c|}{ População residente em zona urbana (\%) } \\
\hline$<50$ & 1,00 & & 1,00 & \\
\hline $50-75$ & 0,83 & $0,83-0,83$ & 0,92 & $0,92-0,92$ \\
\hline$>75$ & 0,46 & $0,46-0,46$ & 0,72 & $0,72-0,72$ \\
\hline \multicolumn{5}{|l|}{ IDH } \\
\hline$<0.6$ & 1,00 & & 1,00 & \\
\hline $0,6-0,7$ & 0,64 & $0,64-0,64$ & 0,77 & $0,77-0,77$ \\
\hline$>0,7$ & 0,39 & $0,39-0,40$ & 0,86 & $0,85-0,86$ \\
\hline \multicolumn{5}{|c|}{ PIB per capita (Reais) } \\
\hline$>10.000$ & 1,00 & & 1,00 & \\
\hline $10.000-20.000$ & 0,76 & $0,76-0,76$ & 0,91 & $0.91-0.91$ \\
\hline$>20.000$ & 0,34 & $0,34-0,34$ & 0.45 & $0.45-0.45$ \\
\hline
\end{tabular}

IC95\%: intervalo de 95\% de confiança; IDH: Índice de Desenvolvimento Humano; OR: odds ratio; PIB: Produto Interno Bruto.

Nota: controlado por: taxa de procedimentos em 2000/2001, porte populacional, ao menos um Centro de Especialidades Odontológicas no município e cobertura de equip de saúde bucal > 80\%.

\section{Tabela 3}

Interação entre a presença de Centro de Especialidades Odontológicas (CEO) e cobertura da equipe de saúde bucal (ESB) sobre a proporção de exodontias em relação aos procedimentos odontológicos preventivos e curativos nos municípios brasileiros em 2015/2016.

\begin{tabular}{lcccccc}
\hline & \multicolumn{3}{c}{ Cobertura da ESB } & \multicolumn{2}{c}{ Interação (valor de $\mathbf{p}$ ) } \\
& OR & IC95\% & OR & IC95\% & \\
\hline Presença de ao menos um CEO & 0,74 & $0,73-0,77$ & 0,71 & $0,71-0,72$ & $<0,01$ \\
\hline
\end{tabular}

IC95\%: intervalo de 95\% de confiança; OR: odds ratio.

Nota: controlado por: Índice de Desenvolvimento Humano, população residente em zona urbana, Produto Interno Bruto per capita, percentual de usuários entre 0 e 19 anos e percentual de usuários mulheres.

Outro importante achado foi a menor proporção de realização de exodontias em municípios com maior IDH, PIB e população residente em zona urbana, com CEO e cobertura de ESB superior a 80\%, observando-se que populações em piores condições socioeconômicas não estão integradas de forma equânime na RASB e, portanto, mais susceptíveis a procedimentos mutiladores em saúde bucal. Esses dados sugerem que a "lei do cuidado inverso" descrito por Hart 9 em nível individual, em que é ofertado mais acesso às pessoas com menos necessidades de saúde, talvez possa ser verdadeiro em nível 
ecológico, em que municípios com mais necessidades (sociais e de saúde) ofertem menos cuidados em saúde. Nessa perspectiva, torna-se importante destacar a necessidade do universalismo proporcional, que sugere que as ações de saúde devem ser universais e em escala e intensidade que estejam de acordo com o nível de desvantagem, podendo ser considerado como uma nova forma de abordagem das políticas públicas de saúde para que alcancem resultados que reduzam também as inequidades em saúde na população 10,11. Com base nisso, sugere-se que se amplie a alocação de recursos no âmbito populacional com o intuito de melhorar o desempenho dos indicadores contextuais que podem impactar na organização e desempenho da RASB e, consequentemente, proporcionar melhores desfechos em saúde bucal ao minimizar a realização de exodontias.

A proporção de exodontias em relação aos procedimentos odontológicos preventivos e curativos é um dos indicadores de avaliação dos serviços de saúde bucal que são realizados nas unidades básicas de saúde ${ }^{2}$. Em uma discussão entre experts em saúde bucal coletiva do Ministério da Saúde, estabeleceu-se a proporção de $8 \%$ de exodontias em relação aos procedimentos ambulatoriais odontológicos. Há estudos descritivos que demonstram a redução de exodontias em relação aos demais procedimentos odontológicos. No Estado do Pará, encontrou-se uma redução na razão entre o número de exodontias e procedimentos odontológicos individuais, de $22,2 \%$ para $17,5 \%$ entre os anos de 2001 e 2010; e um aumento do número de procedimentos da atenção secundária, especialmente de periodontia e endodontia, no período de 2001 a 2010, apesar de não considerar outros fatores, como os contextuais, que podem impactar nestes achados 12 . Outro estudo 13, realizado no Estado do Paraná, avaliou a evolução de procedimentos odontológicos realizados em cinco municípios, entre 2003 e 2009, antes e após a implantação dos CEOs. Os autores encontraram uma tendência de redução no número de exodontias e um aumento de endodontias e de periodontia em três municípios 13. Assim, os achados do presente estudo confirmam em nível nacional a observação de menores proporções de exodontias em relação ao total de procedimentos realizados e demonstra a importância de se revisar o parâmetro estabelecido pelo Ministério da Saúde a fim de estabelecer critérios mais tangíveis que também tenham em conta as particularidades contextuais dos municípios.

Em Santa Catarina, um estudo semelhante foi desenvolvido para avaliar a relação entre dados contextuais e de cobertura odontológica com o indicador de exodontia no contexto da atenção primária em saúde. Os autores também encontraram correlação negativa entre a proporção de exodontias e a cobertura de consultas odontológicas, ou seja, quanto maior a cobertura, menor a proporção de exodontias no município. As cidades com maior IDH também apresentaram melhores resultados 14 . Porém, não existe evidência de pesquisa avaliativa mais abrangente, na perspectiva da rede, apesar de a constituição das redes ser priorizada como estratégia de organização dos sistemas 15 . Essa perspectiva reforça uma fragmentação contrária a RASB que se pretende instituir para ampliar o uso dos serviços odontológicos e garantir a integralidade da atenção. Assim, passa a ser de fundamental importância avaliar o desempenho da RASB e não apenas de pontos como ESB ou CEO de forma isolada, para que os dados apresentados neste estudo confiram a interação entre estes dois pontos da rede, promovendo a integralidade pela oferta de mais procedimentos odontológicos preventivos e curativos em detrimento de exodontias.

A interação entre a presença de CEO e a cobertura superior a 80\% de ESB demonstrou haver menores proporções de exodontias em relação aos procedimentos odontológicos preventivos e curativos. Nesse sentido, a integralidade da RAS tem potencial em produzir melhores desfechos de saúde bucal nos municípios brasileiros, destacando-se que municípios que investem na constituição da RASB têm melhor desempenho, ofertam cuidados com integralidade e, portanto, podem impactar positivamente na saúde bucal de suas populações ${ }^{15}$. Ainda que os municípios possam ofertar atendimentos odontológicos especializados sem obrigatoriamente contar com CEO, os municípios com CEO ofertam mais procedimentos endodônticos e periodontais do que em municípios sem CEO, considerando modelos que incluíram variáveis como tipo e tempo de habilitação do CEO, porte municipal, IDH, PIB per capita e renda média dos habitantes ${ }^{16}$. Ressalta-se, assim, a importância do CEO, como um serviço de saúde fundamental à ampliação da produção ambulatorial de procedimentos curativos e preventivos em odontologia.

Além da cobertura de ESB, destaca-se a importância da organização da RASB pela atenção básica de forma qualificada e resolutiva, o que vai ao encontro de achados de um estudo realizado no Estado do Ceará ${ }^{17}$. No referido estudo, também foram encontrados desafios a serem enfrentados como as 
barreiras geográficas, financeiras e organizacionais que podem estar relacionadas ao baixo aproveitamento de marcação de consultas e uso dos serviços disponibilizados. Em relação à diferença de proporção de exodontias em relação aos procedimentos odontológicos preventivos e curativos entre os anos 2000/2001 e 2015/2016, observa-se que houve uma melhora entre esses períodos, contudo pode demonstrar que a consolidação da RASB ainda não superou o modelo centrado em condições agudas. Um estudo sobre o impacto da ESF na saúde bucal encontrou efeitos positivos em relação ao acesso, ações preventivas coletivas e tratamento de dor de dente, mas, em relação à cobertura de exodontia e tratamentos restauradores, encontrou um efeito negativo ou sem efeito em grande parte dos municípios estudados 18. Possíveis respostas aos desafios relacionados à consolidação da RASB foram descritos em um estudo qualitativo realizado em Florianópolis (Santa Catarina), com entrevistas com gestores, profissionais de saúde e professores graduados em odontologia, e encontrou a implantação da rede regionalizada de atenção à saúde como possível caminho para a potencialização das interações na rede, além de reestruturação do atendimento da atenção primária à saúde para a formalização de fluxos entre os serviços para se alcançar a integração dos mesmos na RASB 19.

Este estudo apresenta as limitações de uma pesquisa ecológica transversal, sem a possibilidade de inferência causal. O viés de informação pode estar presente, visto que o lançamento dos dados é de responsabilidade dos municípios, com possível subnotificação dos procedimentos realizados. Todavia, o processo de coleta foi padronizado e relatado em detalhes, reduzindo as possibilidades de erros sistemáticos. Entretanto, a análise de todo o território nacional e a avaliação inédita do impacto dos CEOs na rede de cuidado em saúde bucal expõem as fortalezas deste estudo.

Pelos resultados inéditos obtidos, conclui-se que nos municípios com CEO e maior cobertura de ESB, houve menor proporção na realização de exodontias em relação a municípios com menores coberturas de ESB nas equipes de saúde da família e sem CEO. Isto sugere que maior oferta conjunta de serviços da atenção primária e secundária contribui para a constituição de um melhor desempenho da RASB, sobretudo em relação à integralidade do cuidado nas ações de saúde bucal com menores proporções de exodontias em relação ao total de procedimentos ofertados à população.

\section{Colaboradores}

C. Stein e F. N. Hugo contribuíram com a concepção e projeto do estudo, interpretação dos dados, redação do artigo, revisão crítica relevante do conteúdo intelectual, aprovação final da versão a ser publicada e são responsáveis por todos os aspectos do trabalho na garantia da exatidão e integridade de qualquer parte da obra. K. W. Santos e A. M. Condessa contribuíram com a concepção e projeto do estudo, interpretação dos dados, redação do artigo, revisão crítica relevante do conteúdo intelectual e aprovação final da versão a ser publicada. R. K. Celeste contribuiu com a concepção e projeto do estudo, análise e interpretação dos dados, redação do artigo, revisão crítica relevante do conteúdo intelectual e aprovação final da versão a ser publicada J. B. Hilgert contribuiu com a concepção e projeto do estudo, interpretação dos dados e aprovação final da versão a ser publicada.

\section{Informações adicionais}

ORCID: Caroline Stein (0000-0003-4777-1630); Karoline Weber dos Santos (0000-0003-05245878); Aline Macarevich Condessa (0000-00028129-4324); Roger Keller Celeste (0000-00022468-6655; Juliana Balbinot Hilgert (0000-00022204-1634); Fernando Neves Hugo (0000-00032222-7719).

\section{Agradecimentos}

C. Stein recebe bolsa de doutorado concedida pela Coordenação de Aperfeiçoamento de Pessoal de Nível Superior (Capes). R. K. Celeste e J. B. Hilgert recebem bolsa PQ-2 do Conselho Nacional de Desenvolvimento Científico e Tecnológico (CNPq). 


\section{Referências}

1. Ministério da Saúde. Diretrizes da Política Nacional de Saúde Bucal. Brasília: Ministério da Saúde; 2004.

2. Ministério da Saúde. Índice de Desempenho do Sistema Único de Saúde (IDSUS). Fichas técnicas dos indicadores. http://idsus.sau de.gov.br/detalhadas.html (acessado em 10/ $\mathrm{Jul} / 2017)$.

3. Ministério da Saúde. A saúde bucal no Sistema Único de Saúde. Brasília: Ministério da Saúde; 2018.

4. Ministério da Saúde. Portaria no 599, de 23 de março de 2006. Define a implantação de Especialidades Odontológicas (CEOs) e de Laboratórios Regionais de Próteses Dentárias (LRPDs) e estabelecer critérios, normas e requisitos para seu credenciamento. Diário Oficial da União 2006; 24 mar.

5. Ministério da Saúde. Sala de apoio à gestão estratégica. Número de CEOs implantados. http://sage.saude.gov.br/\# (acessado em 10/ $\mathrm{Jul} / 2017)$.

6. Ministério da Saúde. Portaria de Consolidação no 5, de 28 de setembro de 2017. Consolidação das normas sobre as ações e os serviços de saúde do Sistema Único de Saúde. Diário Oficial da União 2017; 3 out.

7. Correa GT, Celeste RK. Associação entre a cobertura de equipes de saúde bucal na saúde da família e o aumento na produção ambulatorial dos municípios brasileiros, 1999 e 2011. Cad Saúde Pública 2015; 31:2588-98.

8. Chaves SCL, de Barros SG, Cruz DN, Figueiredo ACL, Moura BLA, Cangussu MCT. Brazilian Oral Health Policy: factors associated with comprehensiveness in health care. Rev Saúde Pública 2010; 44:1005-13.

9. Hart JT. The inverse care law. Lancet 1971; 1:405-12.

10. Marmot M. Fair Society Healthy Lives (The Marmot Review). http://www.instituteofheal thequity.org/resources-reports/fair-societyhealthy-lives-the-marmot-review/fair-soci ety-healthy-lives-full-report-pdf.pdf (acessado em 01/Fev/2019).
11. Carey G, Crammond B, De Leeuw E. Towards health equity: a framework for the application of proportionate universalism. Int J Equity Health $2015 ; 14: 81$.

12. da Silva LS, Santana KR, Pinheiro HHC, do Nascimento LS. Indicators of primary care and specialized oral health care in municipalities in the State of Pará, Brazil: ecological study in the period 2001-2010. Epidemiol Serv Saúde 2013; 22:325-34.

13. da Silva FR, Padilha EZ, Baldani MH. Specialized dental services in medium-sized nonmetropolitan cities in Parana state between 2003 and 2010: an exploratory study. Epidemiol Serv Saúde 2013; 22:641-50.

14. Fernandes LS, Peres MA. Association between primary dental care and municipal socioeconomic indicators. Rev Saúde Pública 2005; 39:930-6.

15. Mendes EV. Health care networks. Ciênc Saúde Colet 2010; 15:2297-305.

16. Celeste RK, Moura FRR, Santos CP, Tovo MF. Análise da produção ambulatorial em municípios com e sem centros de especialidades odontológicas no Brasil em 2010. Cad Saúde Pública 2014; 30:511-21.

17. Pinto VPT, Teixeira AH, Santos PR, de Araujo MWA, Moreira MAG, Saraiva SRM. Evaluation of the accessibility to the Specialized Dental Care Center within the scope of the macro region of Sobral, in the state of Ceara, Brazil. Ciênc Saúde Colet 2014; 19:2235-44.

18. Pereira CRS, Roncalli AG, Cangussu MCT, Noro LRA, Patricio AAR, Lima KC. Impacto da Estratégia Saúde da Família sobre indicadores de saúde bucal: análise em municípios do Nordeste brasileiro com mais de 100 mil habitantes. Cad Saúde Pública 2012; 28:449-62.

19. de Mello ALSF, de Andrade SR, Moyses SJ, Erdmann AL. Oral health care in the health network and the regionalization process. Ciênc Saúde Colet 2014; 19:205-14. 


\section{Abstract}

This study aimed to assess the association between the presence of Specialized Dentistry Centers and dental extractions as a proportion of all dental procedures in public services in Brazil. This was an ecological study that assessed sociodemographic data, oral health services, and outpatient production in 5,333 municipalities in 2000-2001 and 2015-2016. The principal exposure variable was the presence of Specialized Dentistry Centers, and the outcome was the mean national proportion of dental extractions in relation to all preventive and curative dental procedures. Interaction and multiple regression analyses were performed using a binomial model with log link function. The mean national proportions of dental extractions in relation to preventive and curative dental procedures were $27.07 \%$ and $15.11 \%$ in 2000-2001 and 2015-2016, respectively. In the analysis of interaction between the presence of Specialized Dentistry Centers and coverage greater than $80 \%$ by the oral health teams, there were lower proportions of dental extractions in relation to preventive and curative dental procedures $(\mathrm{OR}=0.71 ; 95 \% \mathrm{CI}$ : 0.71-0.72). In the multiple regression analysis, municipalities with Human Development Index of 0.6-0.7 (OR = 0.77; 95\%CI: 0.77-0.77), annual per capita GDP greater than BRL 20,000 (OR = 0.45; 95\% CI: 0.45-045), and proportionally higher urban populations $(O R=0.72$; 95\%CI: 0.72-0.72) showed fewer dental extractions as a proportion of all preventive and curative dental procedures in 2015-2016. In conclusion, there were lower proportions of tooth extractions in municipalities with at least one Specialized Dentistry Center and with a coverage of greater than $80 \%$ by the oral health teams, highlighting that municipalities with a consolidated Oral Health Care Network present better performance in the supply of dental care.

Dental Health Services; Dental Specialties; Health Services Evaluation; Secondary Care; Primary Health Care

\section{Resumen}

Este trabajo tuvo el objetivo de evaluar la asociación entre la presencia de Centros de Especialidades Odontológicas (CEO) y su proporción de exodoncias, en relación con el total de procedimientos odontológicos en los servicios públicos de Brasil. Se trata de un estudio ecológico, en que se evaluaron datos sociodemográficos, servicios de salud bucal $y$ atención ambulatoria en 5.333 municipios, durante los bienios de 2000/2001 y 2015/2016. La principal variable de exposición fue la presencia de CEO y el resultado la media nacional de la proporción de exodoncias, respecto al número de procedimientos odontológicos preventivos y curativos. Se realizó tanto un análisis de interacción, como de regresión múltiple, usando un modelo binomial con función de vinculación logit. La media nacional de la proporción de exodoncias, en relación con los procedimientos odontológicos preventivos y curativos, fue $27,07 \%$ y 15,11\% en 2000/2001 y 2015/2016, respectivamente. En el análisis de interacción entre la presencia de CEO y la cobertura superior a un $80 \%$ de equipos de salud bucal (ESB) hubo menores proporciones de exodoncias, en relación con los procedimientos odontológicos preventivos y curativos (OR =0,71; IC95\%: 0,71-0,72). En el análisis de regresión múltiple, los municipios con un Índice de Desarrollo Humano entre 0, 6-0,7 $(\mathrm{OR}=0,77$; IC95\%: 0,77-0,77), PIB per cápita mayor que BRL 20.000 (OR=0,45; IC95\%: 0,45045) y mayor población residente en zona urbana $(O R=0,72$; IC95\%: 0,72-0,72) presentaron una menor proporción de exodoncias, respecto a los procedimientos odontológicos preventivos y curativos en 2015/2016. Se concluye que hubo menores proporciones de exodoncias, en lo que respecta a los procedimientos odontológicos preventivos y $\mathrm{cu}$ rativos en municipios con al menos un CEO, y con más de un $80 \%$ de cobertura de ESB, lo que apunta a que los municipios con una Red de Atención a la Salud Bucal consolidada tienen un mejor desempeño en la oferta de cuidados odontológicos.

Servicios de Salud Dental; Especialidades Odontológicas; Evaluación de Servicios de Salud; Atención Secundaria de Salud; Atención Primaria de Salud

Recebido em 20/Mar/2019

Versão final reapresentada em 17/Mai/2019

Aprovado em 03/Jun/2019 